\title{
Anagramm und Prosa
}

\author{
Der Maulwurf als Theorie
}

So hängt alles viel enger zusammen als man denkt.

(Eich 1970, 12)

\section{Anagramm und Ironie}

Es scheint klar, dass es sich bei der Prosa um eine Gegebenheitsweise handelt, und zwar nicht von Sprache, sondern in und durch Sprache. Das naturalistische, als strukturalistische Patentlösung beliebte Pendant ist die Narration: das erzählend auf die Reihe und in eine Folge gebrachte minimale Gerüst von Prosa im Stande ihrer proto-grammatischen Noch-nicht-Festgelegtheit - >detensed timer heißt das proto-syntaktische Korrelat (Oaklander und Smith 1994). In dieser proto-strukturell offenen Vor-Gegebenheit gleicht Prosa (sofern man bei Vor-Strukturen überhaupt von Analogien reden kann) dem Anagramm und die Analogie von Anagramm und Prosa ist rhetoriktheoretisch als eine eigentümlich gegenläufige Relation aufgefallen. Frederick Ahl hat sie in der Urmaterie der klassischen lateinischen Literatur bei Ovid und Vergil (und nicht wie Saussure in den davor und darunter liegenden archaischen Restbeständen) als konstitutives Moment aufgewiesen. Die klassischen Anagramme, findet Ahl, ähneln einer vergleichbar un-offensichtlichen, abgründigen Natur von Prosa, von der fraglich ist, ob der rhetorische Befund der Ironie schon auf sie zutrifft: »If Quintilianic irony is the least demonstrable but most acceptable figure of speech, the anagram is without doubt the most demonstrable and least acceptable«, lautet sein Fazit (Ahl 1988, 26). ${ }^{1}$ Quintilian'sche Ironie - die in seinem Handbuch zu unübertroffener Prägnanz gediehene Figur par excellence, in der wie in kaum einer anderen (die Allegorie wäre die einzig relevante andere) die rhetorische Verfassung von Diskursen erfasst ist - ist der Inbegriff dessen, was Prosa in dem etymologischen Urverstand, an den Agamben erinnert hat, ausmacht. Tatsächlich ist es eine >Philosophie der Namen`, die der Prosa als »moteur caché« der Prädikation unterliegt (Coccia 2017, 71). Ironie ist die erste, rhetorisch manifeste Ausbeute der Prosa in

1 Er formuliert dabei die Quintessenz von Ahl 1985; dort ist diese Idee leider kaum fruchtbar gemacht oder durchgehalten; sie muss dem Autor nachträglich aufgegangen sein.

Anselm Haverkamp, NYU New York, LMU München

○ Open Access. (C) 2021 Anselm Haverkamp, publiziert von De Gruyter. (cc)/BY Dieses Werk ist lizenziert unter einer Creative Commons Namensnennung 4.0 International Lizenz.

https://doi.org/10.1515/9783110731569-004 
ihrem proto-typischen Urzustand - und nicht erst ein Effekt der Erzählfiktion, der im ausgewachsenen Roman zu Buche schlägt. Ihre offenbare Verborgenheit, die seit Ramus als >crypsis of method theoretisiert worden ist (Miltons Begriff) und in Baumgartens ramistischer Auffassung als figura cryptica die ästhetische Dimension eröffnet, funktioniert ohne Signale. Oder aber, Ironie der Signale, über verdeckte, verschobene Erkennungszeichen, zu Gattungsmerkmalen geronnene Marken, die als Übermarkierungen von einer überschüssigen, selbstironischen Art sind. Ihre seit Baumgarten fällige ästhetische Theoretisierung hat ein mise en abîme zur Grundfigur, bekommt es mit Sprache als unabsehbarem Abgrund zu tun. Bei Anagrammen verhält es sich - eine Ironie ganz eigener, introvertierter Art - perverserweise umgekehrt.

"Doch bleibt die prosaische Redeweise in formaler Hinsicht unterbestimmt», hat Inka Mülder-Bach $(2019,2)$ die Verlegenheit der Theorie auf den springenden Punkt gebracht. Die Unterbestimmtheit des Überschüssigen liegt vor den gattungspoetischen Begrenzungen, die seit der Konjunktur des Prosabegriffs im neunzehnten Jahrhundert als Gegensatz von Poesie und Prosa hervorgetreten und zur sprichwörtlichen, nachgerade allegorischen Folie sogenannt >prosaischer Lebensverhältnisse geraten sind. Das hat Wolfgang Preisendanz (1968) in einer denkwürdigen Vorlage zum Thema Die nicht mehr schönen Künste auf die Hypothese vom »Funktionsübergang « gebracht, der in der Prosa - Heine ist der paradigmatische Ort (Preisendanz 1973, 22f.) - »Dichtung und Publizistik« verbinde. Der Untertitel des bahnbrechenden Kolloquiums der Poetik und Hermeneutik III über Grenzphänomene des Ästhetischen verrät einen seinerzeit sehr mangelhaften Stand der Theorie und die Provokation des Abweichlers Preisendanz, denn es handelt sich für ihn um kein Grenzphänomen, sondern um einen Grundzug der von Baumgarten auf den Begriff gebrachten, von Kants dritter Kritik vergeblich entschärften, unter dem Begriff der Urteilskraft verharmlosten Aesthetica.

Tatsächlich führt die von Ahl an der Anagrammatik gewonnene Einsicht auf eine weiterhin ungelöste begriffliche Crux der Rhetorik als Grundlage der Poetik, wie sie von Baumgarten revidiert worden ist. Oder wie Paul de Man $(1979,17)$ in seiner Einführung in die Allegories of Reading befand: "Poetic writing is the most advanced and refined mode of deconstruction; it may differ from critical or discursive writing in the economy of its articulation, but not in kind." Poetik - auch die Poetik der Schreibart `Prosa` - bekommt es mit einem Grundzug poetischen Schreibens zu tun, dessen Rhetorizität in der dekonstruktiven Gegebenheit, der Ökonomie der Artikulation, keine Differenzqualität aufweist zur diskursiven, gesprochenen Sprache - weshalb auch Baumgarten nicht zögerte, der Rhetorik, speziell der >crypsis of method ‘ in Gestalt der figurae crypticae von Ironie und Allegorie, ihren rhetorisch angestammten, bewährten Anteil zu lassen. Was macht dann den Unterschied aus, von dem die formale Differenz- 
qualität die Oberfläche ist? De Man legt nahe, »advanced and refined « als Aspekte einer reflektierten Rhetorik aufzufassen; die Gattungen der Poetik also nach der Art >reflexiver Mechanismen` als längerfristig regulierte Vorgaben einer quasi automatisch erbrachten Selbstbezüglichkeit aufzufassen (ich zitiere den nützlichen Begriff Luhmanns, der die sreflexiven Mechanismen nach >Reflexion ` und >Reflexivität ‘ als Momente von >Thematisierung « weiter unterscheidet). ${ }^{2}$

Der Musterfall verdeckter, kryptisch effektiver Ironie in der Fiktion des Romans, wie er in Gestalt von Rousseaus Nouvelle Héloïse de Man vorlag, in der Brieffiktion seit Richardsons Clarissa und, in letzter Steigerung, in den Liaisons dangereuses von Laclos, ist der eines unmerklichen Funktionierens, das für den Moment der Lektüre - genauerhin im Fall der Brieffiktion des Mit-Lesens der Briefe, das quasi über die Schulter der Leser erfolgt - als Roman enthüllt wird (und verfilmbar ist: keine zufällige Konsequenz) (Vinken 1991, 206 ff.; Haverkamp 2018). Die zur Routine gediehene empathisch nachvollziehende Einstellung gegenüber der Romanfiktion nutzt eine Latenz von Prosa aus, bringt sie heraus und zeichnet sie als Latenz der kryptischen Figuration der im Roman qua Prosa mit-thematisch gewordenen Textkonstitution ein. So der in Erinnerung zu bringende Stand der Theorie der siebziger Jahre nach Roland Barthes und de Man. Jean Starobinskis (1971) Rekonstruktion von Saussures AnagrammStudien machte unterhalb der gattungspoetischen Querlage die Prosa als Quelle der im Roman evozierten Latenzen sicht- und fassbar. Romane wie Henry James' What Maisie Knew unterfüttern diese Rolle der Prosa in einer weiteren Verarbeitungsschicht der psychologischen Plausibilisierung; Slavoj Žižek (2006) nennt die lacaneske Raffinierung der kunstfertigen >crypsis` parallaktisch. ${ }^{3}$ Die zunehmende, immer abgründigere Ironie der Romanfiktion von Clarissa bis Finnegans Wake speist sich aus dieser selben Quelle, und Freuds Entdeckung der Latenz, der Saussures Entdeckung der Diachronie passgenau entspricht, gab eine erste Ahnung von der Prosa-Qualität des späten Wake. David Haymans Idee der ınodal structure erhellt den von Joyce eingeschlagenen Mittelweg, auf dem Finnegans Wake im epischen Gewande eine eigenartige ımock-orality، hervorbringt; der Brief der Analphabetin Anna Livia ist das zu Recht bekannteste Stück darin. Indessen, führt Hayman aus, sind die narrativen Reste, die als grammatische Knotenpunkte dem Epos von Finnegans Wake zugrundeliegen, kein Beweis für die originäre Mündlichkeit eines Sängers, sondern sie sind »all cast in the alphabetical mode«, und die sub-literarische Tonalität produziert nichts als ironisch

2 Luhmann 1972, 92-112; sowie weiterführend Luhmann 1975, 72-102.

3 Er bevorzugt The Wings of the Dove. 
kalkulierte side-effects »to the predominantly anti- or meta-narrative discourse of the texts « (Hayman 1990, 11) - ich präzisiere hier: der Prosa. Die Frage, die sich an diesen Befund, der sich weiter differenzieren lässt, anschließt, ist die der anagrammatischen Funktion der sepiphanoiden` Anlage der Prosa, der zwar phänomenal auftretenden, aber zitierend parodierenden alphabetischen materia prima, die in ihrer Wendefähigkeit als Prosa herauspräpariert ist - ein hochartifizielles, jeder originären Anmutung von Schöpfung (die den Joyce-Fans so teuer ist) diametral entgegengesetztes, den Schöpfungsakt Gottes und der selbsternannten Nachfolger im Genie parodierendes Produkt, pure Poiesis auf der Schwelle der paradoxen, »in progress« verzögerten Ins-Werk-Setzung.

»Description, it appears«, so de Man (1986, 51), »was a device to conceal inscription « in nachgerade absoluter >crypsis of figure (davon zehrt auch Blumenbergs ıabsolute Metapher ) oder - Ahls Paradox - phänomenaler Unkenntlichkeit, welche die Ironie aus der Buchstaben-Materie entbindet, entlässt. Der verdeckten anagrammatischen Struktur, die Saussure in der Latenz der Götternamen als archaischen Rest einer verschollenen Diachronie zu entdecken meinte und die Ahl als konstitutives Moment klassischer lateinischer Dichtung erweist, unterliegt eine protogrammatisch-prosaische Schicht von Sprache, als deren später Inbegriff die Prosa der phänomenalen Aus- und Abnutzung derselben Sprache in den Konventionen, Anwendungsroutinen und Inanspruchnahmen von Poesie als gattungspoetische Disziplin entgegentritt. An die Stelle des mythischen Einwirkens benannter, unter göttlichen Namen maskierter Latenz tritt in der Prosa eine aus-wendig er-innerte Leere, die an Leitfäden wie den Namen eine nie und nimmer gemachte Erfahrung in Erlebnissen (neuerlich sevents`) herbeizitiert, und das zu den unterschiedlichsten Zwecken und Vergnügen. Ein >objektiver Humor macht sich breit, den Hegel (ich zitiere Preisendanz (1963)) als die nachklassische Moral von jeder Geschicht' prophezeit. Objektiver Humor statt tiefer gelegter Ironie ist der höhere Zweck und tiefere Sinn der figurae crypticae, die in Prosa verankert sind, aus ihr heraustreten: Anagrammatisch ist die Herkunft ihrer Machart. Beim späteren Joyce liegt sie auf der Hand, beflügelt sie prompt die Leser-Gemeinde, fehlt ihr freilich die Theorie, als deren Masken, wie im Ulysses penetrant, die abgedroschenen Stereotype des Mythos herhalten müssen, angefangen bei dem Titelhelden Odysseus, der zur selben Zeit die Autoren der Dialektik der Aufklärung aufs bürgerliche Glatteis führte (Adorno und Horkheimer 1947, 1. Exkurs). Diese Mythen-Mimikry ging nicht nur Empson auf den Geist, der Finnegans Wake (sic!) als neugotischen Kitsch, »imitation Gothic«, verhöhnte, als wärs ein Pendant des Herrn der Ringe aus dem Hause J.R.R. Tolkiens und Empsons Antagonisten C.S. Lewis' (Empson 1989, $67 \mathrm{ff}$.).

Dabei schreit Prosa im neu entdeckten Sinne nach der nach-klassischen Schreibart, nach dem `Ende der Kunstperiode`, nach begleitender selbstreflexi- 
ver Theorie, wie sie womöglich schon der alten Anagrammatik in impliziter Verborgenheit immanent gewesen sein mag (ein weites Feld, aber man sollte die Möglichkeit nicht unterschätzen). Es ist eine von weither in die Prosa der neueren Gegenwart hineinreichende Ironie der Geschichte, dass die neue Prosa und das angemessene Prosa-Verständnis mit der phänomenalen Verborgenheit der Anagramme und nicht mit dem schlichten phänomenalen Realismus der Romane übereinkommt, und sei es auch nur, dass sie mit ihm konkurrierte (auch das ein Teil desselben weiten Felds). Tatsächlich sind damit die probaten Mittel der Ironisierung erschöpft, die Metaphorologie der Figuren (der Allegorie in Quintilians Verstand) marginalisiert. Selbst Empsons >Ambiguitätı, die post-semantische Symptomatologie der modernen Sprachsituation, scheint nur noch von einer ungeklärten Oberflächen-Relevanz, so wie auch das Fiktionsproblem der mitlaufenden Referenzen bedeutungslos ist. Ein erstes, zwar eher marginales Symptomfeld, das es zu einer eigenen Verlegenheits-Gattung gebracht hat, ist die Kompromissbildung des >Prosagedichts`, deren Theoriehaltigkeit zuerst von Barbara Johnson (1979) ins Auge gefasst und auf den Avantgarde-Befund der >Defiguration` gebracht worden ist. In der Absetzungsbewegung, die schon in den klassischen Misch-Mustern des prosimetrum (von Boethius bis Dante) eine allegorisch ausgefeilte und durchreflektierte Tradition besitzt, stellt das von Johnson als »zweite Revolution« Baudelaires beschriebene Prosagedicht eine mit der klassischen Moderne untergegangene dekonstruktive Schwelle dar (Dronke 1994). Als solche ist sie nicht repräsentativ geworden, sondern artistische Provokation geblieben; die Prosa in ihr ist Poesie oder wie Reinhard Lettau, ein letzter Meister dieser Art von Prosa, so platt wie möglich gesagt hat: „Bei Gedichten werden, wie im vorliegenden/Fall die Zeilen nicht vollgeschrieben « (Lettau 1968, 7). Da sind Günter Eichs Maulwürfe, die späteste Prosa des womöglich besten deutschen Nachkriegslyrikers, von einem anderen Gewicht.

\section{Eich in Ansbach}

Eichs fast schon berühmtes poetologisches Statement, eine in der Form des prosaischen Eingeständnisses listig verkleidete Grundsatzerklärung, ist vertrackter, als es seine ostentative Naivität auf den ersten Blick merken lassen will: »Ich bin über das Dingwort noch nicht hinaus«, bekennt er, um sogleich fortzufahren:

Ich bin über das Dingwort noch nicht hinaus. [...] Ich habe deshalb wenig Hoffnung, einen Roman schreiben zu können. Der Roman hat mit dem Zeitwort zu tun, das im Deutschen mit Recht auch Tätigkeitswort heißt. In den Bereich des Zeitworts bin ich nicht durchgedrungen. Allein das Dingwort brauche ich gewiß noch einige Jahrzehnte.

(Eich 1961, 23-24) 
Das >Dingwort` (Eich zitiert Grundschule) geht auf das rhetorische Doppel res et verba zurück, das als Dingwort eigentümlich treffend übersetzt ist, sofern res die in Rede stehenden nomina spezifiziert: Deren numinose (eine heikle Etymologie) Vorgänger sind, unschwer zu erkennen, die Götternamen der Anagrammatik - keine bloßen Paradigmen, sondern Proto-Paradigmen. Der numinose Grund der nomina lag bis Varro noch auf der Hand, so in seinem Beispiel vom imperium als dictum ab nutu (De lingua latina 7.85). ${ }^{4}$ Das Dingwort nennt, erinnert, wiederholt und liegt dabei vor der Tätigkeit der Zeitwörter; schon Eichs Gedichte liegen vor der erzählenden Sprach-Zeit, in >de-tensed timeく. Sie sind, lässt sich im Nachhinein der Maulwürfe sagen, dezidierte Prosa. In der Rückwendung zur Prosa vollenden sie die von Barbara Johnson an den Petits Poèmes en prose aufgewiesene zweite Revolution, haben sie revolutionären Charakter. Gleichzeitig und im selben Zug - das ist nicht im romantischen Sinne verdoppelter Reflexion selbstverständlich - reflektiert Eichs Maulwurfs-Prosa Theorie, bzw. >zeigtı sie, wie Wittgenstein es sich vorstellte, im Fliegenglas, die ästhetische Konstitution der in Prosa hyperreflexiv wahrnehmbaren Intransparenz als phänomenale Unentscheidbarkeit. Deshalb findet die Fliege den Ausgang nicht; sie kann ihn unmöglich vom Glas unterscheiden.

Ein besonders handliches Beispiel ist der Maulwurf In Ansbach. Er gibt sich vordergründig als Parodie eines städtischen »Faltprospekts«, einer alltäglichen Gattung, die Bedeutsamkeit über die Topologie lokaler Namen produziert. Der touristische Tiefsinn, der parodiert wird, verrät absichtslos, in kaum zu erwartender, schlagartig unerwarteter Dichte, seine tiefere, anagrammatische Bewandtnis. Sie läuft der Grammatik der parodierten Faltblatt-Rhetorik nicht so sehr zuwider, als dass sie das jenseits jeder Absicht sprachlich sedimentierte, dann (erst) rhetorisch mit-transportierte Inschriften-Gewirr heraushebt, das Derrida in Finnegans Wake als shyper-mnesic erkannt hat: eine Mechanik unterhalb der reflexiven Gattungsmechanismen, die den prosaischen Voraussetzungen aufliegt: »a hypermnesic machine capable of storing in an immense epic work Western memory and virtually all the languages in the world including traces of the future." (Derrida 1992, 281) ${ }^{5}$ Der Messianismus der Avantgarden (»including traces of the future« - seine Hervorhebung), der es auch bei Derrida noch mit der Zukunft als Aus- und Erfüllung des virtuell Möglichen aufnimmt, ist meine Sache nicht; im Gegenteil, so scheint mir, erlag Derrida hier dem Geist seiner Zeit, statt - das ıstatt ‘ erkennt er im Sprach-Gedächtnis der Joyce’schen Prosa - der historisch be-

4 Maltby 1991, 416: ad numen. Man vgl. das Genesis-Zitat in Longinus' De sublimitate 9.9 (Haverkamp 2002, Kap. 3).

5 Zuerst erschienen in französischer Sprache: Derrida 1987. 
stimmten Latenz nachzugehen, die in den Phantasmata des Virtuellen abgeschirmt funktioniert. Eich - das macht ihn gegenüber dem Überschwang der Joyceans so einsichtsvoll - widmet sich dem Phantom der alltäglichen Faltprospekthaftigkeit und produziert aus der Implikation der Falten (Eich ist hintergründig, darauf legt er Wert, das weiß man) einen prosaisch, dem Untergrund der Prosa verpflichteten >objektiven Humorı. Die hypermnesische Reflexivität liegt in der eigentümlichen Dichte der schlecht $\mathrm{zu}$ intendierenden, auf Intentionen nicht zuverlässig rückbezüglichen In-schriften, die In Ansbach - hier weht ein letzter Hauch von Ironie an Namen illustriert werden (im Sinne rhetorischer illustratio) und nichts mehr von den Götternamen haben, deren Einschreibung sie nur noch zitieren und örtlich verifizieren, ohne die numinose Herkunft, von der die mickrige Mediokrität der lokalen klassizistischen Epigonen ein groteskes Nachbild zeichnet. Ironie ist in der anagrammatischen Prosa der Maulwürfe die Metapher für den gelöschten, nicht länger - ein Hauch - vorhandenen, ehedem individuellen Anteil an der in Szene gesetzten Objektivität des nun gänzlich >ojektiven Humors`. Ansbach ist kein, nicht einmal ein Spree-Athen. Der Dichte halber, auf deren spezifisch prosaischen Charakter noch zu kommen ist, kann ich nur einen Teil dieses kurzen Textes behandeln - seiner Kürze geradezu zum Trotz.

In Ansbach - Eich tut schon im Titel (stellt sich am Ende heraus) mehr als der Faltprospekt von Ansbach, denn im Namen Ansbach steckt ein Bach, der nicht der Fluss ist, an dem Ansbach liegt. Ich greife hier vor, weil Eich vorgreift. Der Maulwurf In Ansbach fährt nach dieser im Rückblick erkenntlichen Implikation unter dem Titel »In Ansbach« auf das vorerst Argloseste - auch das ein Effekt von Prosa - wie folgt fort:

In Ansbach

In Ansbach entsproß August Graf von Platen Hallermünde, die Tulpe im deutschen Dichtergarten, übrigens in der Platenstraße. Und im Weinmond, am 24. Weinmond 1796, in römischen Ziffern, das macht das Ablesen eindringlich. In einer Parallelstraße entsproß Johann Peter Uz, von dem nicht berichtet wird, welche Blume er darstellt. Er ist auch zufällig in der Uzstraße entsprossen, es ist nicht angegeben in welchem Mond, Rot, Weiß oder Rosé, hoffentlich hat er alle drei getrunken. Auf der Büste im Schloßgarten sieht er lebensfroh in eine Zukunft, die bis 1796 vorhielt.

Die Uzstraße ist nach Hiob benannt, der in Uz entsproß. Mit Gottes Regiment nicht einverstanden, wurde er von Gott gesegnet und ist viel berühmter als sein Heimatort, daher die häufige Verwechslung mit Johann Peter. Man denkt in Ansbach daran, Uz zur Patenstadt zu machen, was kühn aber schwierig wäre, da Johann Peter noch nicht ausgegraben ist.

(Eich 1970, 12) 
Die Dichte der Bezüge ist prosaisch, aber es liegt in der Natur der Prosa, dass aus der prosaischen Dichte intertextuell-semantische Dichte folgt. Ralf Simon orientiert seine Theorie konsequent an Jakobsons Überlagerungsregel, eine vergessene Errungenschaft der Rhetorik Quintilians, die in ihrer Herleitung (vermutlich über Lobecks Dissertationes) ${ }^{6}$ erst unzureichend erkannt und noch unerforscht ist, aber jedenfalls kein Geniestreich, der aus dem Nichts gekommen wäre, sondern aus der Fülle Nach-Baumgarten'scher, Nach-Fontanier'scher, beispielsweise Lobeck'scher Rhetorik-Revisionen. Es ist eine >Verdichtung`, die nach Simon (2014, 129, siehe auch $133 \mathrm{ff}$.) »nicht primär das Ergebnis einer Modellierung durch Form ist « und die als »Matrix« bei Jakobson ein ganzes Spektrum linguistischer Heuristik auf den Plan rief. Der älteren Rhetorik steht sie an verwirrender Theorie-Dichte in nichts nach und die Lütticher Rhétorique générale hat die Beschreibungsdichte auf eine nicht immer fruchtbare Spitze freischwebender, avantgardistischer Bezüge getrieben (die ich hier nicht weitertreiben will). Eichs Maulwürfe bieten statt oder auch zusätzlich zu solchen Bezugs-Phantomen eine angewandte Praxis der ProsaTheorie. Dass dies möglich ist, verdankt sie dem tieferen Widerlager ihrer grammatologischen Disposition, der Prosa, der im rhetorischen Fluss der quasi-narrativ verlaufenden, rhetorisch strukturierten Textentwicklung, die sie ist. Mimetisch heißt bei dem in dieser Frage alles überwölbenden Aristoteles nichts anderes als eingebettet in den von der Rhetorik beherrschten Bereich der »Tätigkeitsworte«, mit deren Syntax - im ısintalkı von Finnegans Wake der mythologischen Besetzungen überführt - Eich es nicht aufnehmen wollte, mit der er nichts zu tun haben wollte (und Beckett hat ihn auch deshalb bewundert). Dichte wäre mithin keine Frage der primär proto-grammatischen Vor-Gegebenheit Prosa, sondern erst und allein der sekundären, syntaktischen Über-Formung, deren >crypsis of method`sie darstellt.

Die sekundären, durchaus auch markierten Ironie-Effekte des Maulwurfs In Ansbach (ob ihrer kalauerhaften Plattheiten berüchtigt) exponieren theoriehaft (auf diesem Wege wird sie theoretisch) ein anagrammatisches Netz von Namen, die für den Fall Ansbach die Struktur der Benennung der Orte und Straßen durch Einschreibung von Heroen und großen Söhnen der Stadt ohne Rücksicht auf Pragmatik oder semantische Pertinenz abbilden. Die illokutionäre Kraft der Prosa »sprengt« also nicht die Syntax, wie Agamben $(2003,23)$ mutmaßt; $^{7}$ sie informiert sie in der Form kryptischer Figuration, und es ist diese UmschlagsWendigkeit, die den Maulwurf ausmacht. Kontingenz ist Spur - »übrigens in

6 Lobeck 1864. Lobeck ist, wenn überhaupt, wegen seiner grammatischen >Pathologien in Erinnerung, in denen man eine Symptomatologie von Prosa erkennen könnte.

7 Zuerst erschienen auf Italienisch, vgl. Agamben 2002. 
der Platenstraße«, »auch zufällig in der Uzstraße«, vom Jahr 1796 noch zu schweigen, das dem Ganzen die Krone aufsetzt (Platens Geburt, Uz' Tod) - bei präzise bezifferter Markiertheit der proto-grammatischen Stelle, not (yet) tensed, aber (und abermals buchstäblich) römisch datiert (in »römischen Ziffern «, »Ablesen eindringlich «) auf die klassische Ära der Anagrammatik, wobei sie deren verunglücktes Weiterleben ganz ironiefrei betrauert. Ich belasse es bei Hinweisen; offenbar verlangt die anagrammatische Vorstruktur der Prosa - ich nenne sie Latenz - als spezifisch ästhetische Errungenschaft eine anwachsende Ausführlichkeit in der Entfaltung der semantischen Folgeerscheinungen. Treffsicher streut Eich ein zufriedenes Fazit ein, eine klassische Gnome, wie er sie liebte (sie war schon die ironie-schwangere Signatur seiner Lyrik), hier also nun aus heiterem Himmel: "So hängt alles viel enger zusammen als man denkt.» (Eich 1970, 12) ${ }^{8}$

Indessen geht der Text nach diesem Satz weiter; das anagrammatische Phantom der Namen, Derridas Metaphern-Phantom ähnelnd, findet zu einem letzten Auftritt, wie im Titel, mit dem »In« vor Ansbach angekündigt (Syntax vor Namen, so etwas wie die Prosa-Regel, die sie ana-grammatisch unterlaufen): "Jetzt habe ich den Ansbacher Bach vergessen« fügt er an und gibt Mnemosyne, der Mutter der Anagrammatik, anstelle der lesmosyne des im Faltprospekt eingefalteten Vergessens, das letzte Wort, binnenreimartig:

Jetzt habe ich den Ansbacher Bach vergessen, die Rezat, für den jährlich die Bachwoche stattfindet, Johann Sebastian Rezat. Damit bin ich so vollständig geworden, daß man sich bei genauer Lektüre den Faltprospekt sparen kann.

$(1970,13)$

Den Streit und die Verrisse, die Eichs Maulwürfe provoziert haben und ihre Leserschaft bis heute spalten, hat ausgerechnet der prosaische Heinrich Böll, wie Beckett ein Eich-Bewunderer der ersten Stunde, unter dem umständlich zutreffenden Titel Flinke, zersetzende, schwer begreifliche, prosaische Maulwürfe überflüssig gemacht: »Obwohl zur Literatur gezählt, sind die Maulwürfe fast keine mehr, ich kenne nichts Vergleichbares, möglicherweise sind sie Ansätze zu einer neuen Philosophie. Ich weiß es nicht.« (Böll 1970, 138f.) In der Tat sind sie das wohl, Ansätze zu einer neuen Philosophie, und Böll hat ihre sokratische Natur im Eingeständnis des wissenden Nicht-Wissens erfasst. Aber nicht Literatur als Philosophie ist die Folge, sondern Philosophie in Literatur: "ihre Zeit«, und zwar in Prosa »erfaßt«, passgenau auf die Diagnose Hegels antwortend, welche die vom

8 Ich verweise hier insgesamt auf meinen älteren Versuch über Lauras Metamorphosen: Dekonstruktion einer lyrischen Figur in der Prosa der Maulwürfe (Haverkamp 1984). Dort, in Eichs Lauren, ist die Gnome »Wenn wir ihren Tod wissen, wissen wir alles.» (Eich 1970, 29) 
>Ende der Kunstperiode begründete. ${ }^{9}$ Eine Dimension von Prosa, die nicht zeitlos prä-historischer, sondern - quasi ana-historisch - gegenwärtiger Art ist.

\section{Literaturverzeichnis}

Adorno, Theodor W. und Max Horkheimer: Dialektik der Aufklärung. Amsterdam 1947. Agamben, Giorgio: Idea della prosa. Macerata 2002.

Agamben, Giorgio: Die Idee der Prosa. Frankfurt a. M. 2003.

Ahl, Frederick: Metaformations. Soundplay and Wordplay in Ovid and Other Classical Poets. Ithaca, NY 1985.

Ahl, Frederick: Ars Est Caelare Artem (Art in Puns and Anagrams Engraved). In: On Puns. The Foundation of Letters. Hrsg. v. Jonathan Culler. Oxford 1988, 17-43.

Böll, Heinrich: Flinke, zersetzende, schwer begreifliche, prosaische Maulwürfe. In: Über Günter Eich. Hrsg. v. Susanne Müller Hanpft. Frankfurt a. M. 1970, 138-139.

Bubner, Rüdiger: Was ist kritische Theorie? In: Hermeneutik und Dialektik. Aufsätze I. Methode und Wissenschaft. Lebenswelt und Geschichte. Hrsg. v. Rüdiger Bubner, Konrad Cramer und Reiner Wiehl. Tübingen 1970, 317-342.

Bubner, Rüdiger: Was ist kritische Theorie? In: Hermeneutik und Ideologiekritik. Hrsg. v. Karl-Otto Apel, Claus v. Bormann, Rüdiger Bubner, Hans-Georg Gadamer, Hans Joachim Giegel und Jürgen Habermas. Frankfurt a. M. 1971, 210-243.

Coccia, Emmanuele: Quodlibet. Logique et physique de l'être quelconque. In: Critique 73 (2017), 66-77.

de Man, Paul: Semiology and Rhetoric. In: ders.: Allegories of Reading. New Haven, CT 1979, 3-19.

de Man, Paul: Hypogram and Inscription. In: ders.: The Resistance to Theory. Minneapolis 1986, 27-53.

Derrida, Jacques: Ulysse gramophone. Deux mots pour Joyce. Paris 1987.

Derrida, Jacques: Ulysses Gramophone. Hear Say Yes in Joyce. In: ders.: Acts of Literature. Hrsg. v. Derek Attridge. New York 1992, 253-309.

Dronke, Peter: Verse with Prose from Petronius to Dante. The Art and Scope of the Mixed Form. Cambridge, MA 1994.

Eich, Günter: Trigonometrische Punkte. In: Mein Gedicht ist mein Messer. Lyriker zu ihren Gedichten. Hrsg. v. Hans Bender. München 1961, $23-24$.

Eich, Günter: Ein Tibeter in meinem Büro. 49 Maulwürfe. Frankfurt a. M. 1970.

Empson, William: The Structure of Complex Words. Cambridge, MA 1989.

Haverkamp, Anselm: Lauras Metamorphosen. Dekonstruktion einer lyrischen Figur in der Prosa der Maulwürfe. In: Deutsche Vierteljahrsschrift für Literaturwissenschaft und Geistesgeschichte 58.2 (1984), 317-346.

9 Hegels Satz aus der Vorrede zur Rechtsphilosophie ist von Rüdiger Bubner (1970) in zeitgemäßer Relevanz, im selben Jahr wie Eichs Maulwurf und am passenden Ort einer GadamerFestschrift kommentiert worden: »Philosophie ist ihre Zeit, in Gedanken erfaßt«. Wiederholt in dem frühen Theorie-Diskussionsband Hermeneutik und Ideologiekritik (Bubner 1971, 230 f.). 
Haverkamp, Anselm: Figura cryptica. Theorie der literarischen Latenz. Frankfurt a. M. 2002. Haverkamp, Anselm: Wirkungsmuster Klopstock. Illusion und Empathie - Die Struktur der teilnehmenden Lektüre in den Leiden Werthers. In: ders.: Klopstock/Milton. Teleskopie der Moderne. Stuttgart 2018, 120-146 [zuerst 1982].

Hayman, David: The 'Wakes in Transit. Ithaca, NY 1990.

Johnson, Barbara: Défigurations du langage poétique. La seconde révolution baudelairienne. Paris 1979.

Lettau, Reinhard: Gedichte. Berlin 1968.

Lobeck, Christian August: Dissertationes de metaphora et de metonymia. Königsberg 1864.

Luhmann, Niklas: Reflexive Mechanismen. In: ders.: Soziologische Aufklärung I. Aufsätze zur Theorie sozialer Systeme. Opladen 1972, 92-112.

Luhmann, Niklas: Selbst-Thematisierung des Gesellschaftssystems. In: ders.: Soziologische Aufklärung II. Aufsätze zur Theorie der Gesellschaft. Opladen 1975, 72-102.

Maltby, Robert: A Lexicon of Ancient Latin Etymologies. Leeds 1991.

Mülder-Bach, Inka: Einleitung. In: Prosa Schreiben. Literatur, Geschichte, Recht. Hrsg. v. Inka Mülder-Bach, Jens Kersten und Martin Zimmermann. Paderborn 2019, 1-11.

Oaklander, L. Nathan und Quentin Smith: Introduction. In: The New Theory of Time. Hrsg. v. L. Nathan Oaklander und Quentin Smith. New Haven, CT 1994, 1-22.

Preisendanz, Wolfgang: Humor als dichterische Einbildungskraft. München 1963.

Preisendanz, Wolfgang: Der Funktionsübergang von Dichtung und Publizistik bei Heine. In: Die nicht mehr schönen Künste. Grenzphänomene des Ästhetischen. Hrsg. v. Hans Robert Jauß. München 1968, 343-374.

Preisendanz, Wolfgang: Der Funktionsübergang von Dichtung und Publizistik. In: ders.: Heinrich Heine: Werkstrukturen und Epochenbezüge. München 1973, 21-68.

Simon, Ralf: Vorüberlegungen zu einer Theorie der Prosa. In: Poetik. Historische Narrative und aktuelle Positionen. Hrsg. v. Armen Avanessian und Jan Niklas Howe. Berlin 2014, 124-144.

Starobinski, Jean: Les mots sous les mots. Les anagrammes de Ferdinand de Saussure. Paris 1971.

Vinken, Barbara: Unentrinnbare Neugierde. Die Weltverfallenheit des Romans. Freiburg i. Br. 1991. Žižek, Slavoj: The Parallax View. Cambridge, MA 2006. 
